\title{
Morse theory for a fourth order elliptic equation with exponential nonlinearity
}

\author{
Laura Abatangelo and Alessandro Portaluri
}

\begin{abstract}
Given a Hilbert space $(\mathcal{H},\langle\cdot, \cdot\rangle)$, and interval $\Lambda \subset(0,+\infty)$ and a map $K \in C^{2}(\mathcal{H}, \mathbb{R})$ whose gradient is a compact mapping, we consider the family of functionals of the type:

$$
I(\lambda, u)=\frac{1}{2}\langle u, u\rangle-\lambda K(u), \quad(\lambda, u) \in \Lambda \times \mathcal{H} .
$$

As already observed by many authors, for the functionals we are dealing with the (PS) condition may fail under just this assumptions. Nevertheless, by using a recent deformation Lemma proven by Lucia (Topol Methods Nonlinear Anal 30(1):113-138, 2007), we prove a Poincaré-Hopf type theorem. Moreover by using this result, together with some quantitative results about the formal set of barycenters, we are able to establish a direct and geometrically clear degree counting formula for a fourth order nonlinear scalar field equation on a bounded and smooth $C^{\infty}$ region of the four dimensional Euclidean space in the flavor of (Malchiodi in Adv Differ Equ 13:1109-1129, 2008). We remark that this formula has been proven with complete different methods in (Lin and Wei Preprint, 2007) by using blow-up type estimates.
\end{abstract}

Mathematics Subject Classification (2000). 35B33, 53A30, 53C21, 58E05.

Keywords. Scalar field equations, Geometric PDE's, Morse Theory, Leray-Schauder degree.

\section{Introduction}

Let $(\mathcal{H},\langle\cdot, \cdot\rangle)$ be a Hilbert space whose associated norm will be denoted by $\|\cdot\|$. Given an interval $\Lambda$ of $(0, \infty)$ and $K$ such that

$$
K \in C^{2}(\mathcal{H}, \mathbb{R}), \quad \text { with } \nabla K: \mathcal{H} \rightarrow \mathcal{H} \text { compact }
$$

\footnotetext{
A. Portaluri was partially supported by PRIN Variational Methods and Nonlinear Differential Equations.
} 
let us consider functionals which are of the form:

$$
I(\lambda, u)=\frac{1}{2}\langle u, u\rangle-\lambda K(u), \quad(\lambda, u) \in \Lambda \times \mathcal{H} .
$$

We observe that the conditions (1)-(2) are not enough to ensure the (PS)-condition which is known to hold only for bounded sequences. (See, [15, Lemma $2.3])$. Therefore the classical flow defined by the vector-field $-\nabla_{u} I(\lambda, u)$ is not suitable to derive a deformation lemma. However, by using a recent deformation result proven by [15, Proposition 1.1], we prove the following result.

Theorem 1. Let $I(\lambda, \cdot)$ be a family of functionals satisfying (1)-(2) and fix $\bar{I}(\cdot):=I(\bar{\lambda}, \cdot)$ for some $\bar{\lambda} \in \Lambda$. Given $\varepsilon>0$, let $\Lambda^{\prime}:=[\bar{\lambda}-\varepsilon, \bar{\lambda}+\varepsilon]$ be a (compact) subset of $\Lambda$ and consider $a, b \in \mathbb{R}(a<b)$, so that all the critical points $\bar{u}$ of $I(\lambda, \cdot)$ for $\lambda \in \Lambda^{\prime}$ satisfy $\bar{I}(\bar{u}) \in(a, b)$. If

$$
\bar{I}_{a}^{b}:=\{u \in \mathcal{H}: a \leq \bar{I}(u) \leq b\},
$$

and assuming that $\bar{I}$ has no critical points at the levels $a, b$, we have

$$
\operatorname{deg}_{L S}\left(\nabla \bar{I}, \bar{I}_{a}^{b}, 0\right)=\chi\left(\bar{I}^{b}, \bar{I}^{a}\right) .
$$

where we denoted by $\operatorname{deg}_{L S}$ the Leray-Schauder degree of the compact vector field $\nabla \bar{I}$.

Now, let $\Omega \subset \mathbb{R}^{4}$ be a bounded smooth domain, and let us consider the following boundary value problem

$$
\begin{cases}\Delta^{2} u=\tau \frac{h(x) e^{u}}{\int_{\Omega} h(x) e^{u} d x} & \text { in } \Omega \\ u=\Delta u=0 & \text { on } \partial \Omega\end{cases}
$$

where $h$ is a $C^{2, \alpha}$ positive function for $\alpha \in(0,1)$, and $\tau \in \mathbb{R}^{+}$. In dimension two and in the second order case the problem has been extensively studied by many authors since the importance of this equation is related to its physical meaning. In fact, it arises in mathematical physics as a mean field equation of Euler flows or for the description of self-dual condensates of some ChernSimons-Higgs model. (See [13-15,6,16], for further details). Moreover semilinear equations involving exponential nonlinearity and fourth order elliptic operators appear naturally in conformal geometry and in particular in prescribing $Q$-curvature on 4-dimensional Riemannian manifolds. (See, for instance [6]).

We denote by $\mathscr{H}$ the space of all functions of Sobolev class $H^{2}(\Omega) \cap$ $H_{0}^{1}(\Omega)$ endowed with the equivalent norm $\|u\|_{\mathscr{H}}:=\|\Delta u\|_{2}$, then problem (4) has a variational structure and for each fixed constant $\tau$, the (weak) solutions can be found as critical points of the functional

$$
I_{\tau}(u):=\frac{1}{2}\|u\|_{\mathscr{H}}^{2}-\tau \log \left(\frac{1}{|\Omega|} \int_{\Omega} h(x) e^{u} d x\right) \quad \forall u \in \mathscr{H},
$$

where we denoted by $|\cdot|$ the Lebesgue measure in $\mathbb{R}^{4}$. The key analytic fact which we need in order to compute the Leray-Schauder degree is a version for higher order operators of the Moser-Trudinger inequality. As a direct consequence of this inequality it follows that the functional (5) is coercive for 
$\tau<64 \pi^{2}$ and thus it is possible to find the solutions of (4), by using the direct method of the calculus of variations. If $\tau>64 \pi^{2}$, the functional $I_{\tau}$ is unbounded both from below and from above and hence the solutions have to be found by other methods, for instance as saddle points, by using some minmax scheme. A general feature of the problem is a compactness property if $\tau$ is not integer multiple of $64 \pi^{2}$ as proven by Lin and Wei in [8].

If $\tau<64 \pi^{2}$ or $\tau \in\left(64 k \pi^{2}, 64(k+1) \pi^{2}\right), k \in \mathbb{N}$, by elliptic regularity and by taking into account the compactness result proven in [10, Theorem 1.2], it is possible to define the Leray-Schauder degree for the boundary value problem (4), fixing a large ball $\mathscr{B}_{R} \subset \mathscr{H}$ centered at 0 and containing all the solutions. In fact, let us consider the family of compact operators $T_{\tau}: \mathscr{B}_{R} \rightarrow \mathscr{H}$, defined by

then the Leray-Schauder degree

$$
T_{\tau}(u):=\tau \Delta^{-2} \frac{h e^{u}}{\int_{\Omega} h e^{u}} ;
$$

$$
d_{\tau}:=\operatorname{deg}_{L S}\left(I-T_{\tau}, \mathscr{B}_{R}, 0\right)
$$

is well-defined for $\tau \neq 64 k \pi^{2}, k \in \mathbb{N}$.

Notation 1. For any two integers $k_{1} \geq k_{2}$, we use the notation $\left(\begin{array}{l}k_{1} \\ k_{2}\end{array}\right)$ to denote

$$
\left(\begin{array}{l}
k_{1} \\
k_{2}
\end{array}\right):= \begin{cases}\frac{k_{1}\left(k_{1}-1\right) \ldots\left(k_{1}-k_{2}+1\right)}{k_{2} !} & \text { if } k_{2}>0 \\
1 & \text { if } k_{2}=0,\end{cases}
$$

and $\mathbf{k}$ to denote the set $\{1, \ldots, k\}$.

By applying Theorem 4.1, together with a precise homological properties of the formal set of barycenters obtained in [6] we can reprove the following result.

Theorem 2. [11] For $\tau \in\left(64 k \pi^{2}, 64(k+1) \pi^{2}\right)$, and $k \in \mathbb{N}$, the Leray-Schauder degree $d_{\tau}$ of (4) is given by

$$
d_{\tau}=\left(\begin{array}{c}
k-\chi(\Omega) \\
k
\end{array}\right),
$$

where $\chi(\Omega)$ denotes the Euler characteristic of the domain $\Omega$.

As a direct consequence if $\chi(\Omega) \leq 0$ then the problem (4) possesses a solution provided that $\tau \neq 64 k \pi^{2}, k \in \mathbb{N}$.

In the rest of the section we briefly describe the method and the main ideas of the proof. As already observed for $\tau>64 \pi^{2}$, the functional $I_{\tau}$ is unbounded both from above and below due to the so-called bubbling phenomenon which often occurs in geometric problems. More precisely, for a given point $x \in \Omega$ and for $\lambda>0$, we consider the following function

$$
\varphi_{\lambda, x}(y)=\log \left(\frac{2 \lambda}{1+\lambda^{2} \operatorname{dist}(y, x)^{2}}\right)^{4}
$$

where $\operatorname{dist}(\cdot, \cdot)$ denotes the metric distance on $\Omega$. For large $\lambda$, one has $e^{\varphi_{\lambda, x}} \rightarrow$ $\delta_{x}$ (the Dirac mass at $x$ ) and moreover one can show that $I\left(\tau, \varphi_{\lambda, x}\right) \rightarrow-\infty$, for 
$\tau \in\left(64 \pi^{2}, 128 \pi^{2}\right)$ as $\lambda \rightarrow+\infty$. Similarly, if $\tau \in\left(64 k \pi^{2}, 64(k+1) \pi^{2}\right)$ for $k>1$, it is possible to construct a function $\varphi$ of the above form (near at each $x_{i}$ ) with $e^{\varphi_{\lambda, \sigma}} \rightarrow \sigma:=\sum_{i=1}^{k} t_{i} \delta_{x_{i}}$ and on which $I_{\tau}$ still attains large negative values. A crucial observation, as proven in [6], is that the constant in Moser-Trudinger inequality can be divided by the number of regions where $e^{u}$ is supported. From this argument we see that one is led naturally to consider the family of elements $\sum_{i=1}^{k} t_{i} \delta_{x_{i}}$ with $\left(x_{i}\right)_{i} \subset \Omega$ and $\sum_{i=1}^{k} t_{i}=1$, known in literature as the formal set of barycenters of $\Omega$ of order $k$ and introduced for the first time by Bahri and Coron in [2]. Using the functions $\varphi_{\lambda, x}$, it is indeed possible to map (non-trivially) this set into $\mathscr{H}$ in such a way that the functional $I_{\tau}$ on the image is close to $-\infty$. On the other hand, it is also possible to do the opposite, namely to map appropriate sublevels of $I_{\tau}$ into the formal set of barycenters. The composition of these two maps turns out to be homotopic to the identity on the formal set of barycenters. We remark that our method is along the same line of a recent result proven by Malchiodi in [17], for a general Paneitz operator on compact four dimensional Riemannian manifolds without boundary.

\section{Preliminaries}

The aim of this section is to recall some abstract results from degree theory for $\alpha$-contractions, Sard's lemma for Fredholm maps and to recall some topological and homological properties of the so-called formal set of barycenters. Our main references will be $[2,5-7,17]$.

The Sard-Smale theorem and Kuratowski non-compactness measure. We start this section with the classical Sard-Smale theorem stated in a form suitable for our purposes. See [5, p. 91].

Theorem 2.1. (Sard-Smale) Let $\Gamma$ be an open subset of a Hilbert space $X$. Suppose that $\mathscr{G} \in C^{1}(\Gamma, X)$ is proper when restricted to any closed bounded subset of $\Gamma$ and that $\nabla \mathscr{G}(x)=\mathrm{Id}-K(x)$ where for every $x \in \Gamma, K(x)$ is a compact operator. Then the set of regular values of $\mathscr{G}$ is dense in $X$.

We will apply this result to $X=\mathscr{H}$ and $\mathscr{G}=\nabla I_{\tau}$. Since both the map $\mathscr{G}$ and its Fréchet derivative are of the form $\mathrm{Id}-K$ where $K$ is a compact operator, than the assumptions of theorem 2.1 are fulfilled.

Now let $\mathscr{F}: \Gamma \rightarrow X$ be a strict $\alpha$-contraction, meaning that $\alpha(\mathscr{F}(B))<$ $k \alpha(B)$ for some fixed $k \in[0,1)$, where $B \subset \Omega$ is a bounded subset and where $\alpha$ denotes the Kuratowski measure of non-compactness. If $y \notin(\operatorname{Id}-\mathscr{F})(\partial \Omega)$ and $(\mathrm{Id}-\mathscr{F})^{-1}(\{y\})$ is compact, we can define the generalized degree Deg, in such a way that if $\mathrm{Id}-\mathscr{F}$ is a compact vector field and $\Gamma$ is a bounded subset it enjoys all the properties of the Leray-Schauder degree.

Formal set of barycenters. The aim of this paragraph is to recall some facts about the formal set of barycenters. 
Following [2], we let $\Omega_{k}$ denote the family of formal sums

$$
\Omega_{k}:=\sum_{i=1}^{k} t_{i} \delta_{x_{i}} ; \quad t_{i} \geq 0, \quad \sum_{i=1}^{k} t_{i}=1 ; \quad x_{i} \in \Omega,
$$

endowed with the weak topology of distributions. This is known in literature as the formal set of barycenters of $\Omega$ of order $k$. We stress the fact that this set is not the family of convex combinations of points in $\Omega$.

In order to give a more topological insight on these spaces, some definitions are in order. We denote by $J_{k}$ the $k$-fold join of $\Omega$. We recall that a point $\underline{x} \in J_{k}$ is specified by:

(i) $k$ real numbers $t_{1}, \ldots t_{k}$ satisfying $t_{i} \geq 0, \sum_{i=1}^{k} t_{i}=1$, and

(ii) a point $x_{i} \in \Omega$ for each $i \in \mathbf{k}$ such that $t_{i} \neq 0$.

Such a point will be denoted by the symbol $\oplus_{i=1}^{k} t_{i} x_{i}$, where the elements $x_{i}$ may be chosen arbitrarily or omitted whenever the corresponding $t_{i}$ vanishes. Furthermore we will endow this space with the strongest topology such that the coordinate functions are continuous. Now, if $\Sigma^{k}$ denotes the symmetric group over $k$ elements, we assume that $\Sigma^{k}$ acts on $J_{k}$ by permuting factors, namely

$$
\forall \sigma \in \Sigma^{k}: \sigma\left(t_{1} x_{1} \oplus \cdots \oplus t_{k} x_{k}\right):=\left(t_{\sigma(1)} x_{\sigma(1)} \oplus \cdots \oplus t_{\sigma(k)} x_{\sigma(k)}\right) .
$$

Thus, the $k$-th symmetric join of $\Omega$, say $S J_{k}$ is defined as the quotient of $J_{k}$ with respect to $\Sigma^{k}$.

Definition 2.2. ([7, Definition 5.1]) The $k$-th barycenter space $\Omega_{k}$ can be defined as the quotient of the symmetric join $S J_{k}$ under the equivalence relation $\sim$ below:

$$
t_{1} x_{1} \oplus t_{2} x_{1} \oplus \cdots \oplus t_{k} x_{k} \sim\left(t_{1}+t_{2}\right) x_{1} \oplus \cdots \oplus t_{k} x_{k} .
$$

That is a point in $\Omega_{k}$ is a formal abelian sum with the topology that when $t_{i}=0$ the entry $0 x_{i}$ is discarded from the sum, and when $x_{i}$ moves in coincidence with $x_{j}$, one identifies $t_{i} x_{i}+t_{j} x_{i}$ with $\left(t_{i}+t_{j}\right) x_{i}$. It is possible to show that we have the embeddings

$$
\Omega \hookrightarrow \Omega_{2} \hookrightarrow \cdots \hookrightarrow \Omega_{k-1} \hookrightarrow \Omega_{k}
$$

and each factor is contractible in the next one. Let $P$ be the projection on $\mathscr{H}$ (i.e. $P \varphi=\varphi-h$ with $\Delta^{2} h=0$ in $\Omega$ and $h=\varphi$ and $\Delta h=\Delta \varphi$ on $\partial \Omega$ ), $\Sigma \subset \mathscr{H}$ be the unit sphere and finally let

$$
R: \mathscr{H} \backslash\{0\} \rightarrow \Sigma: u \mapsto R(u):=u /\|u\|_{\mathscr{H}} .
$$

Let $g_{k}: S J_{k} \rightarrow \Sigma$ be the map defined as: $g_{k}\left(\left(x_{1}, \ldots, x_{k}\right),\left(t_{1}, \ldots, t_{k}\right)\right):=$ $R\left(\sum_{i=1}^{k} t_{i} P \varphi_{\lambda, x_{i}}\right)$, where $\lambda>0$ is fixed and $\varphi_{\lambda, x_{i}}$ are given by

$$
\varphi_{\lambda, x_{i}}(y)=\log \left(\frac{2 \lambda}{1+\lambda^{2} \operatorname{dist}\left(y, x_{i}\right)^{2}}\right)^{4} .
$$

We observe that since two elements in $S J_{k}$ equivalent for the relation introduced in Definition 2.2 have the same image through $g_{k}$, this implies that $g_{k}$ 
is well-defined on the quotient. Denoting by $\Omega^{k}$ the $k$-fold product of copies of $\Omega$ and by $\Delta_{k}$ the collision set $\bigcup_{i, j=1}^{k} \Delta_{i, j}$, where

$$
\Delta_{i, j}:=\left\{\left(x_{1}, \ldots, x_{k}\right) \in \Omega^{k} \mid \quad x_{i}=x_{j}, i \neq j, \text { for } i, j \in \mathbf{k}\right\},
$$

we define the configuration space $\widehat{\mathfrak{X}}_{k}:=\mathfrak{X}_{k} \backslash \Delta_{k}$. Let us consider the fibration

$$
\mu: \widehat{\mathfrak{X}}_{k} \rightarrow \widehat{\mathfrak{X}}_{k-1}, \quad \text { defined by } \mu\left(x_{1}, \ldots, x_{k}\right):=\left(x_{1}, \ldots, x_{k-1}\right),
$$

it is easy to observe that each fiber $\mu^{-1}\left(\left(x_{1}, \ldots, x_{k-1}\right)\right)=\Omega \backslash\left\{x_{1}, \ldots, x_{k-1}\right\}$ is homeomorphic to each other. Thus by using the classical Hopf theorem for fibrations (see, for instance Spanier [18], for further details), the Euler characteristic of $\widehat{\mathfrak{X}}_{k}$ can be computed through the fiber $\Omega \backslash\left\{x_{1}, \ldots, x_{k-1}\right\}$ and $\widehat{\mathfrak{X}}_{k-1}$. By an easy calculations it follows that

$$
\chi\left(\widehat{\mathfrak{X}}_{k}\right)=\chi(\Omega)(\chi(\Omega)-1) \ldots(\chi(\Omega)-k+1) .
$$

Lemma 2.3. (well-known) The set $\Omega_{k} \backslash \Omega_{k-1}$ is an open smooth manifold of dimension $5 k-1$ for each $k \in \mathbb{N}$.

Proof. The case $k=1$ is trivial, since $\Omega_{1}=\Omega$ and $\Omega$ is a four dimensional manifold being an open subset of $\mathbb{R}^{4}$. For $k \geq 2$ the join $J_{k}$ is a smooth manifold. Since the action of the symmetric group on $J_{k}$ is free of fixed points than the symmetric join is a smooth manifold. Moreover, since $\Omega_{k-1}$ is the boundary of $\Omega_{k}$, than $\Omega_{k} \backslash \Omega_{k-1}$ is a smooth open manifold in which the elements in $\Omega_{k} \backslash \Omega_{k-1}$ are smoothly parameterized by $4 k$ coordinates locating the points $x_{i}$ and $k-1$ coordinates identifying the numbers $t_{i}$ 's. The conclusion immediately follows.

Lemma 2.4. (well-known) If $\Omega$ is not contractible, then for any $k \geq 1$ the set $\Omega_{k}$ is a is non contractible stratified set.

Proof. (Sketch). It can be proved by arguing as follows. The case $k=1$ is trivial. For $k \geq 2$ even if the set $\Omega_{k-2}$ is not a smooth manifold (actually it is a stratified set) however it is an ENR which implies that there exists a non trivial ( $\bmod 2)$ orientation class with respect to its boundary. However by using the Čech-cohomology, and by taking into account that it is isomorphic to the singular cohomology and over $\mathbb{Z}_{2}$ to the singular homology, the thesis follows by using the exactness of the pair once it is proven that

$$
H_{5 k-1}\left(\Omega_{k} ; \mathbb{Z}_{2}\right) \simeq H_{5 k-1}\left(\Omega_{k} \Omega_{k-1} ; \mathbb{Z}_{2}\right) .
$$

(See, for instance, [6, Lemma 3.7], for further details).

By using the same arguments as in [17, Proposition 5.1], it can be proven the following result.

Lemma 2.5. Let $\eta$ be positive and let $G:(0,+\infty) \rightarrow(0,+\infty)$ be the nonincreasing function satisfying:

$$
G(t)=\frac{1}{t} \text { for } t \in(0, \eta] \quad G(t)=\frac{1}{2 \eta} \text { for } t>2 \eta .
$$


If $d\left(x_{i}, x_{j}\right):=\operatorname{dist}\left(x_{i}, x_{j}\right)$ and $F^{*}: \Omega_{k} \backslash \Omega_{k-1} \rightarrow \mathbb{R}$ as follows

$$
F^{*}\left(\sum_{i=1}^{k} t_{i} \delta_{x_{i}}\right):=-\sum_{i \neq j} G\left(d\left(x_{i}, x_{j}\right)\right)-\sum_{i=1}^{k} \frac{1}{t_{i}\left(1-t_{i}\right)} .
$$

Then we have

$$
\sum_{i=1}^{5 k-1} c_{i}=(-1)^{k-1} \frac{\chi\left(\widehat{\mathfrak{X}}_{k}\right)}{k !}
$$

where $c_{i}$ denotes the number of critical points of $F^{*}$ of index $i$.

The following result will be crucial in order to compute the Leray-Schauder degree of our result.

Proposition 2.6. For any natural number $k$ we have:

$$
\chi\left(\Omega_{k}\right)=1-\left(\begin{array}{c}
k-\chi(\Omega) \\
k
\end{array}\right) .
$$

Proof. The proof is given by induction over $k$. The case $k=1$ is trivial being $\Omega_{1}$ homeomorphic to $\Omega$. For $k>1$ we consider the pair $\left(\Omega_{k}, \Omega_{k-1}\right)$ and we remark that the Euler characteristic is additive. Thus $\chi\left(\Omega_{k}\right)=\chi\left(\Omega_{k}, \Omega_{k-1}\right)+\chi\left(\Omega_{k-1}\right)$.

Claim 1. The following formula holds for any natural number $k$

$$
\chi\left(\Omega_{k}, \Omega_{k-1}\right)=(-1)^{k-1}\left(\begin{array}{c}
\chi(\Omega)-k \\
k
\end{array}\right) .
$$

Once this is done the proposition easily follows. By Lemma 2.3 the space $\Omega_{k} \backslash \Omega_{k-1}$ is an open manifold of dimension $5 k-1$ with boundary $\Omega_{k-1}$ and by the definition of $F^{*}$, Palais-Smale condition holds.

Observe that $\Omega_{k-1}$ is a deformation retract of the sublevel $F_{-L}^{*}:=\left\{F^{*} \leq\right.$ $-L\} \cup \Omega_{k}$ for $L$ sufficiently large and positive (simply by taking the limit for $L \rightarrow+\infty)$. Thus denoting by $\widehat{F}^{*}:\left\{F^{*} \geq-L\right\} \rightarrow \mathbb{R}$ a non-degenerate function $C^{2}$-close to the restriction of $F^{*}$ to the subset $\left\{F^{*} \geq-L\right\}$, by excision of the sublevel $F_{-L}^{*}:=\left\{F^{*}<-L\right\}$ and by the classical Poincaré-Hopf theorem it holds

$$
\chi\left(\Omega_{k}, \Omega_{k-1}\right)=\sum_{i=1}^{5 k-1}(-1)^{i} c_{i} .
$$

The thesis follows by formula (10) and (8).

Improved Moser-Trudinger inequality. Let $C_{c}^{\infty}(\Omega)$ be the set of all smooth functions with compact support in $\Omega$, and let $\mathscr{H}$ be the completion with respect to the norm $\|u\|_{\mathscr{H}}:=\|\Delta u\|_{2}$. The space $\mathscr{H}$ is a Hilbert space with respect to the scalar product $\langle u, v\rangle_{\mathscr{H}}:=\int_{\Omega} \Delta u \Delta v d x$ for all $u, v \in \mathscr{H}$, and, by the local regularity theorem and by the Poincaré inequality, it follows that $\mathscr{H}$ agrees with the space of all functions on $\Omega$ of Sobolev class $H^{2}(\Omega) \cap H_{0}^{1}(\Omega)$. As immediate consequence of [10, Theorem 1.2] and the Schauder estimates, the following crucial compactness results hold. 
Proposition 2.7. ([10, Theorem 1.2]) Let $h: \Omega \rightarrow \mathbb{R}$ be a positive $C^{2, \alpha}$ function and $\tau \neq 64 k \pi^{2}$ for $k \in \mathbb{N}$. Then the solutions of (4) are bounded in $C^{4, \alpha}(\Omega)$ for any $\alpha \in(0,1)$.

Proposition 2.8. ([8, Lemma 2.1]) Let $u$ be a solution of (4) with $\tau \leq c$, for some constant $c$. Then there exists a $\delta>0$ such that

$$
u(x) \leq c, \quad \forall x \in \Omega_{\delta},
$$

where $\Omega_{\delta}:=\{x \in \Omega: d(x, \partial \Omega) \leq \delta\}$.

We remark that Proposition 2.8 excludes boundary bubbles.

Lemma 2.9. There exists a constant $C_{\Omega}$ depending only on $\Omega$ such that for all $u \in \mathscr{H}$ one has:

$$
\log \left(\frac{1}{|\Omega|} \int_{\Omega} e^{(u-\bar{u})} d x\right) \leq C_{\Omega}+\frac{1}{128 \pi^{2}}\|u\|_{\mathscr{H}}^{2}
$$

where $\bar{u}:=\frac{1}{|\Omega|} \int_{\Omega} u d x$ stands for the average of $u$ over $\Omega$.

Proof. In fact by [1, Theorem 1], there exists $C_{\Omega}^{\prime}>0$ depending only on $\Omega$ such that for all $u \in C_{c}^{2}(\Omega)$ it holds

$$
\frac{1}{|\Omega|} \int_{\Omega} e^{\frac{32 \pi^{2}(u-\bar{u})^{2}}{\|u\|_{\mathscr{H}}^{2}}} d x \leq C_{\Omega}^{\prime}, \quad \forall u \in \mathscr{H} .
$$

Since for every $a, b \in \mathbb{R}$, we have $\left(8 \pi a-\frac{1}{8 \pi} b\right)^{2} \geq 0$ is $2 a b \leq \frac{1}{64 \pi^{2}} b^{2}+64 \pi^{2} a^{2}$, by setting $a:=u-\bar{u}$ and $b=\|u\|_{\mathscr{H}}^{2}$, and exponentiating, we have

$\frac{1}{|\Omega|} \int_{\Omega} e^{(u-\bar{u})} d x \leq e^{\frac{1}{128 \pi^{2}}\|u\|_{\mathscr{H}}^{2}} \frac{1}{|\Omega|} \int_{\Omega} e^{\frac{32 \pi^{2}(u-\bar{u})^{2}}{\|u\|_{\mathscr{H}}^{2}}} d x \leq e^{\frac{1}{128 \pi^{2}}\|u\|_{\mathscr{H}}^{2}} C_{\Omega}^{\prime}, \quad \forall u \in \mathscr{H}$.

Taking the logarithm of this last inequality the conclusion follows by setting $C_{\Omega}:=\log C_{\Omega}$.

In order to study how the function $e^{u}$ is spread over $\Omega$ we need some quantitative results. In fact, we will show that if $e^{u}$ has integral bounded from below on $(l+1)$-regions, the constant $\frac{1}{128 \pi^{2}}$, can be basically divided by $(l+1)$. The proof of the proposition 2.10, is up to minor modifications, an adaptation of the arguments given in [6, Lemma 2.2]; we will reproduce it for the sake of completeness.

Proposition 2.10. For any fixed integer $l$, let $\Omega_{1}, \ldots, \Omega_{l+1}$ be subsets of $\Omega$ satisfying dist $\left(\Omega_{i}, \Omega_{j}\right) \geq \delta_{0}$, for $i \neq j$, when $\delta_{0}$ be positive real number, and let $\gamma_{0} \in\left(0, \frac{1}{l+1}\right)$. Then for any $\tilde{\varepsilon}>0$ there exists a constant $\widetilde{C}:=\widetilde{C}\left(l, \tilde{\varepsilon}, \delta_{0}, \gamma_{0}\right)$ such that

$$
\log \left(\frac{1}{|\Omega|} \int_{\Omega} e^{(u-\bar{u})} d x\right) \leq \frac{1}{128(l+1) \pi^{2}-\tilde{\varepsilon}}\|u\|_{\mathscr{H}}^{2}+\widetilde{C},
$$

for all functions $u \in \mathscr{H}$ satisfying

$$
\frac{\int_{\Omega_{i}} e^{u} d x}{\int_{\Omega} e^{u} d x} \geq \gamma_{0} \quad \forall i \in \mathbf{l}+\mathbf{1}
$$


Proof. We consider $(l+1)$ smooth cut-off functions $g_{1}, \ldots, g_{l+1}$, satisfying the following properties:

$$
\begin{cases}g_{i}(x) \in[0,1] & \text { for every } x \in \Omega ; \\ g_{i}(x)=1 & \text { for every } x \in \Omega_{i}, i \in \mathbf{1}+\mathbf{1} \\ g_{i}(x)=0 & \text { if } \operatorname{dist}\left(x, \Omega_{i}\right) \geq \frac{\delta_{0}}{4} \\ \left\|g_{i}\right\|_{C^{4}(\Omega)} \leq C_{\delta_{0}}, & \end{cases}
$$

where $C_{\delta_{0}}$ depends only on $\delta_{0}$. By interpolation, (see, for instance, [12, Prop. 4.1]), for any $\varepsilon>0$, there exists $C_{\varepsilon, \delta_{0}}$, such that for any $v \in \mathscr{H}$, and for any $i \in \mathbf{1}+\mathbf{1}$ there holds

$$
\left\|g_{i} v\right\|_{\mathscr{H}}^{2}:=\int_{\Omega}\left|\Delta\left(g_{i} v\right)\right|^{2} d x \leq \int_{\Omega} g_{i}^{2}|\Delta v|^{2} d x+\varepsilon \int_{\Omega}|\Delta v|^{2} d x+C_{\varepsilon, \delta_{0}} \int_{\Omega} v^{2} d x
$$

Let $u-\bar{u}=u_{1}+u_{2}$ with $u_{1} \in L^{\infty}(\Omega)$, then from our assumptions we deduce

$$
\begin{aligned}
\int_{\Omega_{i}} e^{u_{2}} d x & \geq e^{-\left\|u_{1}\right\|_{L^{\infty}(\Omega)}} \int_{\Omega_{i}} e^{(u-\bar{u})} d x \\
& \geq e^{-\left\|u_{1}\right\|_{L^{\infty}(\Omega)}} \gamma_{0} \int_{\Omega} e^{(u-\bar{u})} d x, \quad i \in \mathbf{l}+\mathbf{1} .
\end{aligned}
$$

By invoking inequality (12) in lemma 2.9, together with the last two inequalities, it follows that

$$
\begin{aligned}
\log \left(\frac{1}{|\Omega|} \int_{\Omega} e^{(u-\bar{u})} d x\right) & \leq \log \frac{1}{\gamma_{0}}+\left\|u_{1}\right\|_{L^{\infty}(\Omega)}+\log \left(\frac{1}{|\Omega|} \int_{\Omega} e^{g_{i} u_{2}} d x\right)+C_{\Omega} \\
& \leq \log \frac{1}{\gamma_{0}}+\left\|u_{1}\right\|_{L^{\infty}(\Omega)}+\frac{1}{128 \pi^{2}}\left\|g_{i} u\right\|_{\mathscr{H}}^{2}+C_{\Omega} .
\end{aligned}
$$

We choose $i$ such that $\int_{\Omega}\left|\Delta\left(g_{i} u_{2}\right)\right|^{2} d x \leq \int_{\Omega}\left|\Delta\left(g_{j} u_{2}\right)\right|^{2} d x$, for each $j \in \mathbf{l}+\mathbf{1}$. Since the functions $g_{j}$ have disjoint supports, the last formula and (15), implies that

$$
\begin{aligned}
\log \left(\frac{1}{|\Omega|} \int_{\Omega} e^{(u-\bar{u})} d x\right) \leq & \log \frac{1}{\gamma_{0}}+\left\|u_{1}\right\|_{L^{\infty}(\Omega)}+C_{\Omega} \\
& +\left(\frac{1}{128(l+1) \pi^{2}}+\varepsilon\right)\left\|u_{2}\right\|_{\mathscr{H}}^{2}+C_{\varepsilon, \delta_{0}} \int_{\Omega} v^{2} d x .
\end{aligned}
$$

Now let $\lambda_{\varepsilon, \delta_{0}}$ to be an eigenvalue of $-\Delta^{2}$ such that $\frac{C_{\varepsilon, \delta_{0}}}{\lambda_{\varepsilon, \delta_{0}}}<\varepsilon$, and we set

$$
u_{1}:=P_{V_{\varepsilon, \delta_{0}}}(u-\bar{u}) ; \quad u_{2}:=P_{V_{\varepsilon, \delta_{0}}^{\perp}}(u-\bar{u}) .
$$

Here $V_{\varepsilon, \delta_{0}}$ is the direct sum of the eigenspaces of $-\Delta^{2}$ with Navier boundary conditions and having eigenvalues less or equal than $\lambda_{\varepsilon, \delta_{0}}$ and $P_{V_{\varepsilon}, \delta_{0}}, P_{V_{\varepsilon, \delta_{0}}^{\perp}}$ the orthogonal projections onto $V_{\varepsilon, \delta_{0}}$ and $V_{\varepsilon, \delta_{0}}^{\perp}$, respectively. Since $V_{\varepsilon, \delta_{0}}$ is finite dimensional, the $L^{2}$ norm and $L^{\infty}$ norm of $u-\bar{u}$ on $V_{\varepsilon, \delta_{0}}$ are equivalent; then, by using the Poincaré-Wirtinger inequality (cfr. [3, p. 308]), there holds:

$$
\left\|u_{1}\right\|_{L^{\infty}(\Omega)}^{2} \leq \hat{C}_{\varepsilon, \delta_{0}}\left\|u_{1}\right\|_{L^{2}(\Omega)}^{2} \leq \hat{C}_{\varepsilon, \delta_{0}}\left\|u_{1}\right\|_{\left(H^{2} \cap H_{0}^{1}\right)(\Omega)}^{2} \leq \hat{C}_{\varepsilon, \delta_{0}}^{\prime}\left\|u_{1}\right\|_{\mathscr{H}}^{2}
$$


where $C_{\varepsilon, \delta_{0}}^{\prime}$ is another constant depending only on $\varepsilon$ and $\delta_{0}$. Furthermore

$$
C_{\varepsilon, \delta_{0}} \int_{\Omega} u_{2}^{2} d x \leq \frac{C_{\varepsilon, \delta_{0}}}{\lambda_{\varepsilon, \delta_{0}}}\left\|u_{2}\right\|_{H^{2}(\Omega) \cap H_{0}^{1}(\Omega)}^{2} \leq \varepsilon\left\|u_{2}\right\|_{H^{2}(\Omega) \cap H_{0}^{1}(\Omega)}^{2} \leq \varepsilon C_{\Omega}^{\prime}\left\|u_{2}\right\|_{\mathscr{H}}^{2},
$$

where $C_{\Omega}^{\prime}$ is a constant depending only on $\Omega$. Hence the last formulas imply

$$
\begin{aligned}
\log \left(\frac{1}{|\Omega|} \int_{\Omega} e^{(u-\bar{u})} d x\right) \leq & \log \frac{1}{\gamma_{0}}+C_{\varepsilon, \delta_{0}}^{\prime}\left\|u_{1}\right\| \mathscr{H}+C_{\Omega} \\
& +\left(\frac{1}{128(l+1) \pi^{2}}+\varepsilon\right)\left\|u_{2}\right\|_{\mathscr{H}}^{2}+\varepsilon C_{\Omega}^{\prime}\left\|u_{2}\right\|_{\mathscr{H}} \\
\leq & \log \frac{1}{\gamma_{0}}+C_{\Omega}+\left(\frac{1}{128(l+1) \pi^{2}}+3 \varepsilon\right)\|u\|_{\mathscr{H}}^{2}+\bar{C}_{\varepsilon, \delta_{0}}
\end{aligned}
$$

where $\bar{C}_{\varepsilon, \delta_{0}}$ depends only on $\varepsilon$ and $\delta_{0}$ (and $l$ which is fixed). This concludes the proof.

In the next Lemma we show a criterion which implies the situation described in the first condition in (13).

Lemma 2.11. ([6, Lemma 2.3]) Let $l$ be a given positive integer, and suppose that $\varepsilon$ and $r$ are positive numbers. Suppose that for a non-negative function $f \in L^{1}(\Omega)$ with $\|f\|_{1}=1$ there holds

$$
\int_{\cup_{i=1}^{l} B_{r}\left(p_{i}\right)} f d x<1-\varepsilon, \quad \forall l-\text { tuple } p_{1}, \ldots, p_{l} \in \Omega .
$$

Then there exists $\bar{\varepsilon}>0$ and $\bar{r}>0$, depending on $\varepsilon, r, l$ and $\Omega$ (but not on $f$ ), and $l+1$ points $\bar{p}_{1}, \ldots, \bar{p}_{l+1} \in \Omega$ satisfying

$$
\int_{B_{\bar{r}}\left(\bar{p}_{1}\right)} f d x \geq \bar{\varepsilon}, \ldots, \int_{B_{\bar{r}}\left(\bar{p}_{l+1}\right)} f d x \geq \bar{\varepsilon} ; \quad B_{2 \bar{r}}\left(\bar{p}_{i}\right) \cap B_{2 \bar{r}}\left(\bar{p}_{j}\right)=\emptyset \quad \text { for } i \neq j .
$$

Lemma 2.12. If $\tau \in\left(64 k \pi^{2}, 64(k+1) \pi^{2}\right)$ with $k \geq 1$, the following property holds. For any $\varepsilon>0$ and any $r>0$ there exists a large positive $L=L(\varepsilon, r)$ such that for every $u \in \mathscr{H}$ with $\frac{1}{|\Omega|} \int_{\Omega} e^{u} d x=1$ and $I_{\tau}(u) \leq-L$ there exist $k$ points $p_{1, u}, \ldots, p_{k, u} \in \Omega$ such that

$$
\frac{1}{|\Omega|} \int_{\Omega \backslash \cup_{i=1}^{k} B_{r}\left(p_{i, u}\right)} e^{u} d x<\varepsilon
$$

Proof. To prove the thesis, we argue by contradiction. Thus, there exist $\varepsilon, r>0$ and a sequence $\left(u_{n}\right)_{n} \in \mathscr{H}$ with $1 /|\Omega| \int_{\Omega} e^{u_{n}} d x=1$ and $I_{\tau}\left(u_{n}\right) \rightarrow-\infty$ such that for every $k$-tuple $p_{1}, \ldots, p_{k}$ in $\Omega$ we have $1 /|\Omega| \int_{\Omega \backslash \cup_{i=1}^{k} B_{r}\left(p_{i}, u\right)} e^{u} d x \geq \varepsilon$. Now applying Lemma 2.11 with $l=k, f=e^{u_{n}}$ and finally with $\delta_{0}=2 \bar{r}, \Omega_{j}=$ $B_{\bar{r}}\left(\bar{p}_{j}\right)$ and $\bar{\gamma}_{0}=\bar{\varepsilon}$ for $j \in \mathbf{k}$ and where the symbols $\delta_{0}, \Omega_{j}, \bar{\gamma}_{0}$ were defined in Lemma 2.9 and $\bar{r}, B_{\bar{r}}\left(\bar{p}_{j}\right), \bar{\varepsilon},\left(\bar{p}_{j}\right)_{j}$ were defined in Lemma 2.11. By this it follows that, for any given $\tilde{\varepsilon}>0$ there exists a constant $C>0$ depending on $\varepsilon, \tilde{\varepsilon}$ and on $r$ such that

$$
I_{\tau}\left(u_{n}\right) \geq \frac{1}{2}\left\|u_{n}\right\|_{\mathscr{H}}^{2}-C \tau-\frac{\tau}{64(k+1) \pi^{2}-\tilde{\varepsilon}} \frac{1}{2}\|u\|_{\mathscr{H}}^{2},
$$


where the constant $C$ does not depends on $n$. Now since $\tau<64(k+1) \pi^{2}$, we can choose $\tilde{\varepsilon}>0$ small enough that the number $1-\frac{\tau}{64(k+1) \pi^{2}-\tilde{\varepsilon}}:=\delta^{\prime}>0$. Therefore the inequality (17) reduces to

$$
I_{\tau}\left(u_{n}\right) \geq \frac{\delta^{\prime}}{2}\left\|u_{n}\right\|_{\mathscr{H}}^{2}-C \tau \geq-K
$$

where $K$ is a positive constant independent of $n$. This violates our contradiction assumption, and conclude the proof.

Given a non-negative $L^{1}$ function $f$ on $\Omega$, we define the distance of $f$ from $\Omega_{k}$ as

$$
\operatorname{dist}\left(f, \Omega_{k}\right):=\sup \left\{\left|\int_{\Omega} f \psi d x-\langle\sigma, \psi\rangle\right|: \sigma \in \Omega_{k}, \text { and }\|\psi\|_{C^{1}(\Omega)} \leq 1\right\},
$$

where we denoted by $\langle\cdot, \cdot\rangle$ the usual duality product. We also define the set

$$
\mathscr{D}_{\varepsilon, k}=\left\{f \in L^{1}(\Omega): f \geq 0,\|f\|_{L^{1}(\Omega)}=1, d\left(f, \Omega_{k}\right)<\varepsilon\right\} .
$$

With this notation in mind, by Lemma 2.12 we deduce the following.

Lemma 2.13. Suppose $\tau \in\left(64 k \pi^{2}, 64(k+1) \pi^{2}\right)$ with $k \geq 1$. Then for any $\varepsilon>0$ there exists a large positive $L=L(\varepsilon)$ such that for all $u \in \mathscr{H}$ with $I(\tau, u) \leq-L$ and $1 /|\Omega| \int_{\Omega} e^{u} d x=1$, we have $\operatorname{dist}\left(e^{u}, \Omega_{k}\right)<\varepsilon$.

We remark that as a direct consequence of [10, Theorem 1.2,(ii)], the blow-up points $p_{j, u}$ at which the local-mass is concentrated cannot lie on the boundary of $\Omega$.

\section{A topological argument}

The aim of this section is to show that an image of the $\Omega_{k}$ can be mapped into very negative sublevels of the Euler functional $I_{\tau}$. Moreover if $\Omega$ is non contractible then this map is non-trivial in the sense that it carries some homology. The goal of this section is to sketch the proof of the following result which is given along the lines of [16].

Proposition 3.1. For any $k \in \mathbb{N}$ and $\tau \in\left(64 k \pi^{2}, 64(k+1) \pi^{2}\right)$, there exists $L>0$ such that the sublevel $\mathscr{H}^{-L}$ has the same homology as $\Omega_{k}$.

The proof of the Proposition 3.1 will follows from the homotopy invariance of the homology groups once the following facts will be established.

Mapping $\boldsymbol{\Omega}_{\boldsymbol{k}}$ into very low sublevels of $\boldsymbol{I}_{\boldsymbol{\tau}}$. To do so, for $\eta>0$ small enough, consider the smooth non-decreasing cut-off function $\chi_{\eta}: \mathbb{R}^{+} \rightarrow \mathbb{R}$ satisfying the following properties:

$$
\begin{cases}\chi_{\eta}(t)=t, & \text { for } t \in[0, \eta] \\ \chi_{\eta}(t)=2 \eta, & \text { for } t \geq 2 \eta \\ \chi_{\eta}(t) \in[\eta, 2 \eta], & \text { for } t \in[\eta, 2 \eta]\end{cases}
$$


Then given $\sigma \in \Omega_{k}, \lambda>0$ and $\delta>0$ as in proposition 2.8, we can define a smooth function $\tilde{\varphi}_{\lambda, \sigma}: \Omega \rightarrow \mathbb{R}$ such that in $\Omega \backslash \Omega_{\delta} \cup \Omega_{\delta / 2}$ it is given by:

$$
\tilde{\varphi}_{\lambda, \sigma}(y):= \begin{cases}\varphi_{\lambda, \sigma}(y) & \text { for } y \in \Omega \backslash \Omega_{\delta} \\ 0 & \text { for } y \in \Omega_{\delta / 2},\end{cases}
$$

for

$$
\varphi_{\lambda, \sigma}(y):=\log \sum_{i=1}^{k} t_{i}\left(\frac{2 \lambda}{1+\lambda^{2} \chi_{\eta}^{2}\left(d_{i}(y)\right)}\right)^{4}
$$

with $d_{i}(y):=d\left(y, x_{i}\right)$.

Proposition 3.2. Let $\tilde{\varphi}_{\lambda, \sigma}$ be defined above. Then as $\lambda \rightarrow+\infty$ the following properties hold

(i) $e^{\varphi_{\lambda, \sigma}} \rightarrow \sigma$ weakly in the sense of distributions;

(ii) $I_{\tau}\left(\tilde{\varphi}_{\lambda, \sigma}\right) \rightarrow-\infty$ in $\mathscr{H}$ uniformly with respect to $\sigma \in \Omega_{k}$.

Proof. To prove $(i)$ we first consider the function

$$
\bar{\varphi}_{\lambda, x_{i}}(y):=\left(\frac{2 \lambda}{1+\lambda^{2} \chi_{\eta}^{2}\left(d_{i}(y)\right)}\right)^{4}, \quad \forall y \in \Omega,
$$

where $x$ is a fixed point in $\Omega$. It is easy to verify that $\bar{\varphi}_{\lambda, x_{i}}(y) \rightarrow \delta_{x_{i}}$ for $\lambda \rightarrow+\infty$. Then $(i)$ follows from the explicit expression of $\varphi_{\lambda, \sigma}$.

In order to prove (ii), we evaluate separately each term of $I_{\tau}$, and claim that the following estimates hold

$$
\begin{aligned}
& \log \left(\frac{1}{|\Omega|} \int_{\Omega} e^{\tilde{\varphi}_{\lambda, \sigma}} d x\right)=O(1) \quad \text { as } \lambda \rightarrow+\infty \\
& \left.\frac{1}{2}\left\|\tilde{\varphi}_{\lambda, \sigma}\right\|_{\mathscr{H}}^{2} \leq\left(128 k \pi^{2}+o_{\epsilon}(1)\right) \log \lambda+C_{\epsilon}\right) \quad\left(\text { uniformly in } \sigma \in \Sigma_{k}\right),
\end{aligned}
$$

where $o_{\epsilon}(1) \rightarrow 0$ as $\epsilon \rightarrow 0$ and where $C_{\epsilon}$ is a constant independent $\left(x_{i}\right)_{i}$.

The proof of (20) is easy and it follows by integrating over $\Omega$. The proof of (21) is much more involved and it follows by arguing as in [6, Lemma 4.2].

Mapping very low sublevels of $\boldsymbol{I}_{\boldsymbol{\tau}}$ into $\boldsymbol{\Omega}_{\boldsymbol{k}}$ and an homotopy inverse. The main idea is to construct a non-trivial continuous map $\psi: \mathscr{H} \rightarrow \Omega_{k}$ from the sublevels of the Euler functional into $\Omega_{k}$ such that the composition $\psi \circ \phi_{\lambda}$ is homotopic to identity on $\Omega_{k}$.

Proposition 3.3. Suppose that $\tau \in\left(64 k \pi^{2}, 64(k+1) \pi^{2}\right)$ with $k \geq 1$. Then there exists $L>0$ and a continuous projection $\psi: \mathscr{H}^{-L} \rightarrow \Omega_{k}$ with the following properties.

(i) If $\left(u_{n}\right)_{n} \subset \mathscr{H}^{-L}$ is such that $e^{u_{n}} \rightarrow \sigma$, for some $\sigma \in \Omega_{k}$, then $\psi\left(u_{n}\right) \rightarrow \sigma$;

(ii) if $\tilde{\varphi}_{\lambda, \sigma}$ is as above, then for any $\lambda$ sufficiently large the map $\sigma \mapsto \psi\left(\tilde{\varphi}_{\lambda, \sigma}\right)$ is homotopic to the identity on $\Omega_{k}$. 
Proof. First of all we observe that item $(i)$ follows directly from item (ii) and Proposition 3.2. The non-trivial part is the construction of the global continuous projection map $\psi$ which is a left homotopy inverse has proven in [6, Sect. 3].

We close this section by observing that, up to minor modifications, the above defined map $\psi$ is also a right inverse homotopy as proven in $[17$, Appendix]. Thus summing up we conclude that

Corollary 3.4. Given $L$ sufficiently large the topological spaces $\mathscr{H}^{-L}$ and $\Omega_{k}$ are equivalent, up to homotopy.

\section{A Poincaré-Hopf Theorem without Palais-Smale}

The aim of this section is to prove an analogous of the Poincaré-Hopf theorem for a special class of functionals. To do so, let $(\mathcal{H},\langle\cdot, \cdot\rangle)$ be a Hilbert space whose associated norm will be denoted by $\|\cdot\|$. Given an interval $\Lambda$ of $(0, \infty)$ and a map $K$ such that

$$
K \in C^{2}(\mathcal{H}, \mathbb{R}), \quad \text { with } \nabla K: \mathcal{H} \rightarrow \mathcal{H} \text { compact, }
$$

let us consider the functionals which are of the form:

$$
I(\lambda, u)=\frac{1}{2}\langle u, u\rangle-\lambda K(u), \quad(\lambda, u) \in \Lambda \times \mathcal{H} .
$$

It is well-known (see, for instance, [15, Lemma 2.3]) that the conditions (22)(23) could not be enough to ensure the (PS)-condition which is known to hold only for bounded sequences. Now by using the deformation Lemma proven in [15, Proposition 1.1], we are in position to derive the following result.

Theorem 4.1. (A Poincaré-Hopf theorem) Let $I(\lambda, \cdot)$ be a family of functionals satisfying $(22)-(23)$ and fix $\bar{I}(\cdot):=I(\bar{\lambda}, \cdot)$ for some $\bar{\lambda} \in \Lambda$. Given $\varepsilon>0$, let $\Lambda^{\prime}:=[\bar{\lambda}-\varepsilon, \bar{\lambda}+\varepsilon]$ be a (compact) subset of $\Lambda$ and consider $a, b \in \mathbb{R}(a<b)$, so that all the critical points $\bar{u}$ of $I(\lambda, \cdot)$ for $\lambda \in \Lambda^{\prime}$ satisfy $\bar{I}(\bar{u}) \in(a, b)$. Assuming that $\bar{I}$ has no critical points at the levels $a, b$, we have

$$
\operatorname{deg}_{L S}\left(\nabla \bar{I}, \bar{I}_{a}^{b}, 0\right)=\chi\left(\bar{I}^{b}, \bar{I}^{a}\right) .
$$

The proof of this result will be given into two main steps. In the first step we will assume that all the critical points are non-degenerate; in the second step we will remove this assumption.

Proof. First step: non-degenerate case. We let $\mathscr{K}$ denote the set of critical points of $\bar{I}$ which is compact by hypothesis. By compactness and nondegeneracy assumptions, $\bar{I}$ has only finitely-many critical levels each of whose consists only of finitely-many critical points. Let $R$ be so large that all the critical points of $I_{\lambda}$ for $\lambda \in \Lambda^{\prime}$ are in $\mathscr{B}_{\frac{R}{2}}(0)$. Then we can define the cut-off function $\theta: \mathcal{H} \rightarrow[0,1]$ satisfying

$$
\theta(u)=1 \text { for } u \in \mathcal{B}_{R}(0) ; \quad \theta(u)=0 \text { for } u \in \mathcal{H} \backslash \mathcal{B}_{2 R}(0) .
$$


Following Lucia in [15], let $Z \in C^{1}(\mathcal{H}, \mathcal{H})$ be defined by:

$$
Z(u):=-[|\nabla K(u)| \nabla \bar{I}(u)+|\nabla \bar{I}(u)| \nabla K(u)],
$$

and choose $\omega_{\varepsilon} \in C^{\infty}(\mathbb{R})$ such that

$$
0 \leq \omega_{\varepsilon} \leq 1, \quad \omega_{\varepsilon}(\zeta)=0 \quad \text { for all } \zeta \leq \varepsilon, \quad \omega_{\varepsilon}(\zeta)=1 \quad \text { for all } \zeta \geq 2 \varepsilon .
$$

Finally we can define

$$
W(u):=-\omega_{\varepsilon}\left(\frac{|\nabla \bar{I}(u)|}{|\nabla K(u)|}\right) \nabla \bar{I}(u)+Z(u),
$$

where $\omega_{\varepsilon}(|\nabla \bar{I}(u)| /|\nabla K(u)|)$ is understood to be equal 1 when $\nabla K(u)=0$. Given the vector field:

$$
\widetilde{W}(u):=-\theta(u) \nabla \bar{I}(u)+(1-\theta(u)) W(u),
$$

we observe that it decreases $\bar{I}$ in the complement of $\mathscr{K}$. We consider the local flow $\eta=\eta\left(t, u_{0}\right)$ defined by the Cauchy problem:

$$
\frac{d u}{d t}=\widetilde{W}(u), \quad u(0)=u_{0} .
$$

Claim 1. If $\bar{I}$ has no critical levels inside some interval $[\tilde{a}, \tilde{b}]$, then the sub-level $\bar{I}^{\tilde{a}}$ is a deformation retract of $\bar{I}^{\tilde{b}}$.

To prove this, we argue as follows. Given $u_{0} \in \bar{I}^{\tilde{b}}$, we can prove that

$$
\bar{I}\left(\eta\left(t, u_{0}\right)\right) \leq-c^{2} t+\bar{I}\left(u_{0}\right) \cdot{ }^{1}
$$

Thus there exists a $t$ such that $\bar{I}\left(\eta\left(t, u_{0}\right) \leq \tilde{a}\right.$. Then we define:

$$
t_{\tilde{a}}\left(u_{0}\right):= \begin{cases}\inf \left\{t \geq 0: \bar{I}\left(\eta\left(t, u_{0}\right)\right) \in \bar{I}^{\tilde{a}}\right\} & \text { if } \bar{I}\left(u_{0}\right)>\tilde{a} \\ 0 & \text { if } \bar{I}\left(u_{0}\right) \leq \tilde{a} .\end{cases}
$$

Now the map

$$
\widetilde{\eta}:[0,1] \times \mathcal{H} \rightarrow \mathcal{H}, \quad\left(s, u_{0}\right) \mapsto \eta\left(s t_{\tilde{a}}\left(u_{0}\right), u_{0}\right),
$$

is a deformation retraction, as required.

Now let $\bar{c}_{i}$ be the number of critical points of $\bar{I}$ of index $i$. By classical Morse-theoretical arguments as in [4, Theorem 3.2, 3.3, pagg. 100-103], by excising $\{\bar{I}<\tilde{a}\}$, it follows that

$$
\operatorname{deg}_{L S}\left(\nabla \bar{I}, \bar{I}_{a}^{b}, 0\right)=\sum_{i}(-1)^{i} \bar{c}_{i}=\chi\left(\bar{I}^{b}, \bar{I}^{a}\right) .
$$

This concludes the proof in the non degenerate case.

Second step: degenerate case. We reduce ourselves to the non-degenerate case. To do so, fix a small $\delta>0$ so that $\operatorname{dist}\left(\mathscr{K}, \bar{I}_{a}^{b}\right)>4 \delta$, and define the set $\mathscr{K}_{\delta}=\{u \in \mathcal{H}: \operatorname{dist}(u, \mathscr{K})<\delta\}$. We next choose a smooth cut-off function $p$ such that

$$
p(u)=1 \text { for every } u \in \mathscr{K}_{\delta} ; \quad p(u)=0 \text { for every } u \in \mathcal{H} \backslash \mathscr{K}_{2 \delta} .
$$

\footnotetext{
${ }^{1}$ The proof of this inequality is the most involved part of this claim and it can be proven up to minor modifications repeating word by word the arguments given in [15, pp. 121-122].
} 
We can also choose $p$ such that $0 \leq p(u) \leq 1$ for all $u \in \mathcal{H}$ and having uniformly bounded derivative in $\mathscr{K}_{2 \delta}$. Now let $\mathscr{G}:=\left.\nabla \bar{I}\right|_{\mathscr{K}_{\delta}}: \mathscr{K}_{\delta} \rightarrow \mathcal{H}$. Since the map $\mathscr{G}$ is a compact perturbation of the identity, by applying the SardSmale theorem (see Theorem 2.1), the set of regular values of $\mathscr{G}$ is dense in $\mathcal{H}$. This implies that we can find an arbitrarily small $u_{0}$ such that $\nabla \mathscr{G}(p)$ is non-degenerate for each $p \in \mathscr{G}^{-1}\left(u_{0}\right)$ which is equivalent to say that $\nabla^{2} \bar{I}$ is non-degenerate on the set

$$
\Gamma\left(u_{0}\right):=\left\{u \in \mathcal{H}: \nabla I(u)=u_{0}\right\} \cap \mathscr{K}_{\delta} .
$$

Moreover we observe that $\|\nabla \bar{I}\| \geq \gamma_{\delta}>0$ on $\mathscr{K}_{2 \delta} \backslash \mathscr{K}_{\delta}$ for some constant $\gamma_{\delta}$. Now let us consider the function

$$
\widetilde{I}(u):=\bar{I}(u)+p(u)\left\langle u_{0}, u\right\rangle .
$$

It can be shown that the following facts hold:

(i) $\widetilde{I}$ coincides with $\bar{I}$ in $\mathcal{H} \backslash \mathscr{K}_{2 \delta}$;

(ii) $\widetilde{I}$ has the same critical points as $I(\tau, \cdot)$ in $\mathscr{H} \backslash \mathscr{K}_{\delta}$;

(iii) $\widetilde{I}$ is non-degenerate in $\bar{I}_{a}^{b}$.

Item (i) is trivial. To prove (ii) we observe that since $\widetilde{I}$ and $\bar{I}$ coincides out of $\mathscr{K}_{2 \delta}$, it is enough to prove the claim for $u \in \overline{\mathscr{K}_{2 \delta}} \backslash \mathscr{K}_{\delta}$. By differentiating, we have

$$
\langle\nabla \widetilde{I}(u), v\rangle=\left\langle\nabla \bar{I}(u)+\nabla p(u)\left\langle u, u_{0}\right\rangle+p(u) u_{0}, v\right\rangle, \quad \forall v \in \mathcal{H} .
$$

Thus, by recalling that $u \in \overline{\mathscr{K}_{2 \delta}} \backslash \mathscr{K}_{\delta}$, it follows that

$$
\begin{aligned}
& \|\nabla \widetilde{I}(u)\| \geq\|\nabla \bar{I}(u)\|-\mid\left\langle u, u_{0}\right|\|\nabla p(u)\|-p(u)\left\|u_{0}\right\| \\
& \quad \geq \gamma_{\delta}-\left\|u_{0}\right\|(\|\nabla p(u)\|\|u\|+1)>0
\end{aligned}
$$

where the last inequality follows since $p$ has uniformly bounded derivatives and $u_{0}$ can be chosen arbitrarily small. To prove (iii) we argue as follows. Since all the critical points of $\widetilde{I}$ are in $\mathscr{K}_{\delta}$, let us assume by contradiction that $\widetilde{I}$ is degenerate at some critical point $\bar{u}$. Now since $\bar{u} \notin \mathscr{K}$, it follows that $\bar{u} \in \mathscr{K}_{\delta} \backslash \mathscr{K}$. Moreover $\nabla \widetilde{I}(\bar{u})=0$ is equivalent to say that $\nabla \bar{I}(\bar{u})=u_{0}$ and therefore $\bar{u} \in \Gamma\left(u_{0}\right)$. But this is contradict the fact that $\nabla^{2} \widetilde{I}(p)$ is non-degenerate on $p \in \Gamma\left(u_{0}\right)$.

Now, for $\left\|u_{0}\right\|$ sufficiently small the map $\nabla I-I d$ is a strict $\alpha$-contraction. (See Sect. 2) and since $(\nabla \widetilde{I})^{-1}\left(\left\{u_{0}\right\}\right)=\mathscr{K}$, the generalized degree $\operatorname{Deg}(\nabla \widetilde{I}$, $\left.\bar{I}_{a}^{b}, u_{0}\right)$ is well-defined; moreover it coincides with $\operatorname{Deg}\left(\nabla \widetilde{I}, \bar{I}_{a}^{b}, 0\right)$ since it is locally constant. With the above choice for $R$ and by using the excision property and the homotopy invariance of the generalized degree, (see, for instance, [5] for further details), we have

$$
\operatorname{deg}_{L S}\left(\nabla \tilde{I}, \mathscr{B}_{R}, 0\right)=\operatorname{Deg}\left(\nabla \widetilde{I}, \mathscr{B}_{R}, 0\right) .
$$

Now choosing a possibly larger $R$ in such a way $\mathscr{K}_{2 \delta} \subset \mathscr{B}_{R / 2}$, the conclusion readily follows by the first step, simply by replacing $\bar{I}$ with $\widetilde{I}$. 
Corollary 4.2. If $\tau \in\left(64 k \pi^{2}, 64(k+1) \pi^{2}\right)$ for some $k \in \mathbb{N}$ and if $b$ is sufficiently large positive, the sub-level $\mathscr{H}^{b}$ is a deformation retract of $\mathscr{H}$ and hence it has the homology of a point.

Proof. This result follows, by using the deformation constructed in the proof of the Poincaré-Hopf theorem. See, for instance [17, Corollary 2.8].

Setting

$$
J(u):=\log \left(\frac{1}{|\Omega|} \int_{\Omega} h(x) e^{u} d x\right)
$$

the functional (5) can be put in the following form: $I_{\tau}(u)=\frac{1}{2}\|u\|_{\mathscr{H}}^{2}-\tau J(u)$.

\section{Proof of Theorem 2.}

Proof. In order to prove Theorem 2, it is enough to apply Theorem 4.1 to the functional (5) for $\lambda=\tau, \Lambda=\left(64 k \pi^{2}, 64(k+1) \pi^{2}\right)$ for $k \geq 1, \mathcal{H}=\mathscr{H}$ and finally $K(u)=J(u)$ where $J$ was given in (26). The only thing it should be noted, is that all the critical points $\bar{u}$ of $I_{\tau}$ for $\tau \in[\bar{\tau}-\varepsilon, \bar{\tau}+\varepsilon] \subset\left(64 k \pi^{2}, 64(k+1) \pi^{2}\right)$ satisfy $\bar{I}(\bar{u}) \in(a, b)$. This is a consequence of Proposition 2.7 and of the boundedness of $J$ which is consequence of the Moser-Trudinger inequality. Now the conclusion follows choosing $a=-L$ as in Proposition 3.1 and $b$ as in Corollary 4.2. In fact by using Theorem 4.1, we have that

$$
d_{\tau}=\chi\left(\bar{I}^{b}, \bar{I}^{a}\right)=\chi\left(\bar{I}^{b}\right)-\chi\left(\bar{I}^{a}\right)=\chi(\mathscr{H})-\chi\left(\Omega_{k}\right)=1-\chi\left(\Omega_{k}\right) .
$$

The conclusion follows by invoking Proposition 2.6.

Remark 4.3. We observe that the Leray-Schauder degree in the Sobolev space $\mathscr{H}$ coincides with the degree in every Hölder space $C^{2, \alpha}(\Omega), \alpha \in(0,1)$. See for instance [9, Part I, Appendix B, Theorem B.1-B.2].

\section{Acknowledgements}

We would like to thank Prof. A. Malchiodi for suggesting the problem and for many useful discussions about this research project.

\section{References}

[1] David R., Adams: A sharp inequality of J. Moser for higher order derivatives. Ann. Math. 128(2), 385-398 (1988)

[2] Bahri, A., Coron, J.M.: On a nonlinear elliptic equation involving the critical Sobolev exponent: the effect of the topology of the domain. Commun. Pure Appl. Math. 41, 253-294 (1988)

[3] Brezis, H.: Analisi Funzionale. Teoria e Applicazioni. Serie di Matematica e Fisica. Liguori Editore

[4] Chang, K.C.: Infinite-dimensional Morse Theory and Multiple Solution Problems PNLDE 6. Birkhaüser Boston Inc., Boston (1993) 
[5] Deimling, K.: Nonlinear Function Analysis. Springer, Berlin (1985)

[6] Djadli, Z., Malchiodi, A.: Existence of conformal metrics with constant Q-curvature. Ann. Math. (2) 168(3), 813-858 (2008)

[7] Kallel, S., Karoui, R.: Symmetric joins and weighted barycenters. ArXiv:math/ 0602283v2

[8] Lin, C., Wei, J.: Sharp estimates for bubbling solutions of a fourth order mean field equation. Ann. Sc. Norm. Super. Pisa Cl. Sci. (5) 6(4), 599-630 (2007)

[9] Li, Y.Y.: Prescribed scalar curvature on $S^{n}$ and related problems. Part I. J. Differential Equations 120(2), 319-410 (1995). Part II. Existence and compactness. Commun. Pure Appl. Math. 49(6), 541-597 (1996)

[10] Lin, C., Wei, J.: Locating the peaks of solutions via the maximum principle. II. A local version of the method of moving planes. Commun. Pure Appl. Math. 56(6), 784-809 (2003)

[11] Lin, C., Wei, J., Wang, L.: Topological Degree of fourth order mean field equations. Math. Z. (2007)

[12] Lions, J.-L.: Équations différentielles opérationnelles et problèmes aux limites. In: Die Grundlehren der mathematischen Wissenschaften, Bd. 111, 292 p. Springer, Berlin (1961)

[13] Lucia, M.: A mountain pass theorem without Palais-Smale condition. C.R. Acad. Sci. Paris, Ser. I 341, 287-291 (2005)

[14] Lucia, M.: A blowing-up branch of solutions for a mean field equation. Calc. Var 26(3), 313-330 (2006)

[15] Lucia, M.: A deformation Lemma with an application to a mean field equation. Topol. Methods Nonlinear Anal. 30(1), 113-138 (2007)

[16] Malchiodi, A.: Topological methods for an elliptic equation with exponential nonlinearities. Discrete Contin. Dyn. Syst. 21(1), 277-294 (2008)

[17] Malchiodi, A.: Morse theory and a scalar field equation on compact surfaces. Adv. Differ. Equ. 13, 1109-1129 (2008)

[18] Spanier, E.H.: Algebraic Topology. Mc-Graw-Hill, New York (1966)

L. Abatangelo

University of Milano-Bicocca, Milano, Italy

e-mail: l.abatangelo@campus.unimib.it

A. Portaluri

University of Salento, Lecce, Italy

e-mail: alessandro.portaluri@unisalento.it

Received: 23 December 2009.

Revised: 09 May 2010.

Accepted: 07 June 2010. 